\title{
Feshbach resonances in a quasi-two-dimensional atomic gas
}

\author{
M. Wouters, J. Tempere, ${ }^{*}$ and J. T. Devreese ${ }^{\dagger}$ \\ TFVS, Universiteit Antwerpen, Universiteitsplein 1, 2610 Antwerpen, Belgium
}

(Received 10 May 2003; published 3 November 2003)

\begin{abstract}
Strongly confining an ultracold atomic gas in one direction to create a quasi-two-dimensional system alters the scattering properties of this gas. We investigate the effects of confinement on Feshbach scattering resonances and show that strong confinement results in a shift in the position of the Feshbach resonance as a function of the magnetic field. This shift, as well as the change of the width of the resonance, is computed. We find that the resonance is strongly damped in the thermal gas, but in the condensate the resonance remains sharp due to many-body effects. We introduce a two-dimensional model system, suited for the study of resonant superfluidity and having the same scattering properties as the tightly confined real system near a Feshbach resonance. Exact relations are derived between measurable quantities and the model parameters.
\end{abstract}

DOI: 10.1103/PhysRevA.68.053603

\section{INTRODUCTION}

The ability to tune the interatomic interaction strength by using a Feshbach resonance has led to renewed interest in the scattering theory of cold trapped atoms, in particular, in relation with the realization of resonant superfluidity of fermionic atoms [1]. Also the ability to confine Bose-Einstein condensates in periodic potentials has attracted much interest recently, since the resulting optical lattices are ideally suited to probe fundamental quantum effects such as the superfluid to Mott insulator transition [2]. One-dimensional optical lattices allow us to create stacks of "pancake"-shaped clouds filled with neutral atoms [3-5], where an effectively twodimensional (2D) regime can be achieved. In this paper, we address a central question on the crossroads between the two aforementioned achievements, Feshbach scattering and optical lattices: what happens to the Feshbach resonance as the atoms are strongly confined and become (quasi-) twodimensional?

In Sec. II, we derive the 2D resonant scattering amplitude in terms of quantities that can be measured in experiments on $3 \mathrm{D}$ gases. For many-body calculations, it can be helpful to replace the complex real atomic interaction by a simpler effective interaction. To achieve this, we investigate in Sec. III a schematic model of coupled atoms and molecules that interact through a contact potential. Such model systems already have been used before in three dimensions [6], but the connection between the model parameters and the experimental quantities in two dimensions is not yet available. By matching the scattering properties of this model to the results from Sec. II, we derive expressions for the strengths of the effective contact interactions as a function of experimentally measurable (and tunable) quantities. This solves the problem of determining the parameters of a Friedberg-Lee-like Hamiltonian [7] for atomic gases. Such models have been studied in the context of high temperature superconductivity, but for these systems fixing the model parameters remained a problem.

\footnotetext{
*Also at Lyman Laboratory of Physics, Harvard University, Cambridge, MA 02138, USA.

${ }^{\dagger}$ Also at T.U.E., P.B. 513, 5600MB Eindhoven, The Netherlands.
}

PACS number(s): 03.75.-b, 32.80.Pj, 05.30.Fk, 05.30.Jp

\section{QUASI-2D RESONANT SCATTERING}

The existence of a Feshbach resonance relies on the presence of a bound state in a closed channel, with an energy equal to the energy of the colliding particles. In the case of trapped cold atoms, the closed channels involve states with higher Zeeman energy in the external magnetic field. The energy of the bound state (molecule) is tunable by changing this magnetic field. Because these molecular states are small compared to the length scale of the confinement potential, they are not expected to be influenced strongly by the trapping potential. However, this is only a simplified picture. Because of the coupling between the molecular state and the open channels, the binding energy of the molecule acquires a complex shift (a change of the binding energy and a decay width). This shift depends on the nature of the open channels and consequently on the tight confinement.

If the external confinement potential varies slowly on the scale of the atomic interaction range, the two-body scattering can be treated analytically, even in the vicinity of a Feshbach resonance (see also Ref. [8]). In the following, we denote the range of the van der Waals interatomic potential with $R_{e}$ and the oscillator length of the tight confinement in the axial direction by $\ell_{z}=\sqrt{\hbar /\left(2 m_{\text {red }} \omega_{z}\right)}$, with $m_{\text {red }}$ $=\left(m_{1}^{-1}+m_{2}^{-1}\right)^{-1}$ as the reduced mass of the scattering atoms and $\omega_{z}$ the characteristic frequency of the trap in the axial direction. For interatomic distances $R_{e} \ll r \ll \ell_{z}$, both the interatomic and the confinement potential are negligible compared to the total energy and the asymptotic $s$-wave scattering wave function is consequently that for free 3D motion

$$
\psi(\mathbf{r}) \propto \frac{\sin \left(q r+\delta_{3 \mathrm{D}}(E)\right)}{q r},
$$

with $q=\sqrt{2 m_{\text {red }} E} / \hbar$. The energy $E$ is measured in the center of mass frame of the scattering atoms, relative to the energy of two atoms in the open channel at infinity. The phase shift $\delta_{3 \mathrm{D}}(E)$ is determined by the solution of the free 3D scattering problem. At a Feshbach resonance, this phase shift shows resonant behavior, but the wave function (1) remains valid. Close to the resonance, the energy dependence of $\delta_{3 \mathrm{D}}(E)$ is 
important, because it characterizes the resonance. From the analytic theory of Feshbach resonances [9] it follows that it is given by

$$
\delta_{3 \mathrm{D}}(E)=\delta_{b g}-\arctan \left[\frac{\Gamma_{F} / 2}{E-E_{F}-\Delta_{F}}\right],
$$

where $\delta_{b g}$ is the 3D "background" scattering phase shift related only to the open channels, and $\Gamma_{F}, E_{F}$, and $\Delta_{F}$ are the width and the position of the 3D Feshbach resonance, respectively, and the shift of that position due to the coupling with the open scattering channels.

Next, we look at large interatomic distances $r \gg R_{e}$, where the motion is governed by the confining potential and must be a solution of

$$
\left[-\frac{\hbar^{2}}{2 m_{\mathrm{red}}} \nabla^{2}+\frac{1}{2} m_{\mathrm{red}} \omega_{z}^{2} z^{2}\right] \psi(\mathbf{r})=E \psi(\mathbf{r}) .
$$

This problem was analyzed in Refs. [10,11]. Using their solution of Eq. (3) and matching it for $r \rightarrow 0$ to Eq. (1) we find for the scattering amplitude

$$
f_{2 \mathrm{D}}(E)=\frac{2 \sqrt{2 \pi}}{-q \ell_{z} \cot \delta_{3 \mathrm{D}}(E)+\frac{1}{\sqrt{2 \pi}}\left[\ln \left(\frac{C \hbar \omega_{z}}{\pi E}\right)+i \pi\right]},
$$

where $\delta_{3 \mathrm{D}}(E)$ is given by Eq. (2) and $C=0.915$ [11]. Making use of formulas derived in Ref. [9], $\cot \delta_{3 \mathrm{D}}(E)$ can be related to measurable quantities

$$
\cot \delta_{3 \mathrm{D}}(E)=-\frac{1}{q a_{b g}} \frac{E-\left[B-B_{0}+\Delta B\left(q a_{b g}\right)^{2}\right] \Delta \mu}{E-\left(B-B_{0}-\Delta B\right) \Delta \mu},
$$

where $B_{0}$ and $\Delta B$ are the $3 \mathrm{D}$ experimental position and width of the Feshbach resonance, respectively, and $a_{b g}$ is the background scattering length. $\Delta \mu=\mu_{1}+\mu_{2}-\mu_{r e s} ; \mu_{1,2}$ are the atomic magnetic moments and $\mu_{\text {res }}$ is the magnetic moment of the resonant state.

Formula (5), together with Eq. (4) gives the 2D resonant scattering amplitude for a quasi-2D situation in terms of parameters that can be measured in 3D experiments. It should be noted that for low energies (small compared to $E_{F}$ $+\Delta_{F}$, which is typically of the order $\mu_{B} \times 1 \mathrm{G}=k_{B}$ $\times 67.17 \mu \mathrm{K}$, where $\mu_{B}$ is the Bohr magneton), the energy dependence of Eq. (5) is weak and $\cot \delta_{3 \mathrm{D}}(E)$ is well approximated by its value for $E=0$, namely $-q \ell_{z} \cot \delta_{3 \mathrm{D}}(E)$ $\rightarrow \ell_{z} / a_{3 \mathrm{D}}$, which would yield the usual relation $a_{3 \mathrm{D}}$ $=a_{b g}\left[1-\Delta B /\left(B-B_{0}\right)\right]$ for a Feshbach resonance in three dimension.

A remarkable feature of the expression (4) is that it shows that in two dimensions the scattering amplitude never diverges, because $\left|f_{2 \mathrm{D}}(E)\right|^{2} \leqslant 16$, irrespective of $E$ or $E_{F}$. This is in contrast with the $3 \mathrm{D}$ case where the imaginary part in the denominator of the scattering amplitude $\left(=\Gamma_{F}\right)$ is proportional to $\sqrt{E}$ and vanishes as $E \rightarrow 0$ [9]. However, when many-body effects are taken into account for bosonic atoms, the damped character of the Feshbach resonance in two di-

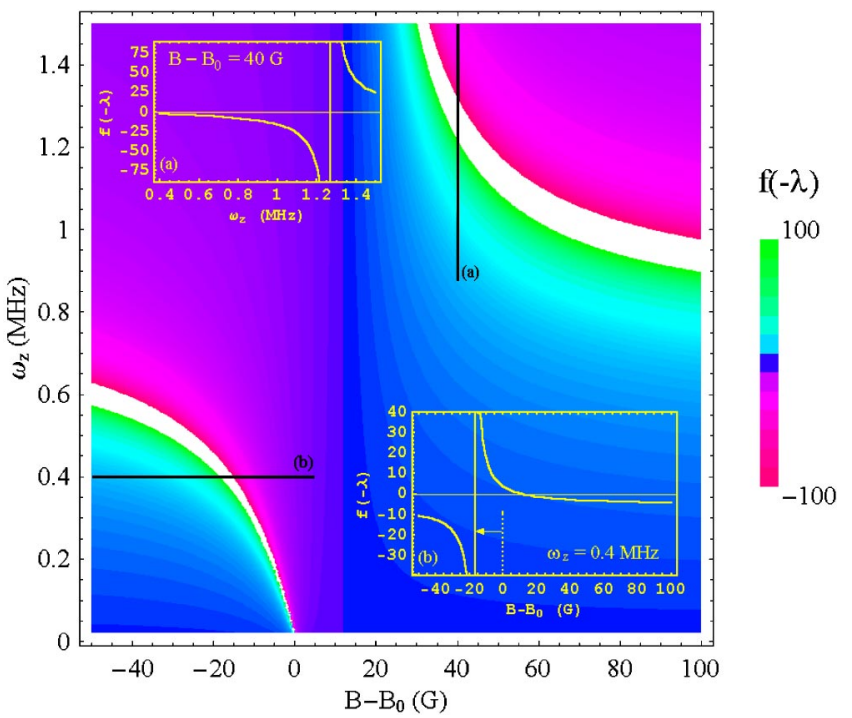

FIG. 1. (Color online) The scattering amplitude $f_{2 \mathrm{D}}(-\lambda)$ for a quasi-2D ${ }^{85} \mathrm{Rb}$ condensate, with chemical potential $\lambda / \hbar=5 \mathrm{kHz}$, is shown color-coded as a function of the magnetic field and the trapping frequency in the tightly confined direction $\omega_{z}$, close to the $3 \mathrm{D}$ Feshbach resonance at $B_{0}=154.9 \mathrm{G}$. The white regions correspond to $|f(-\lambda)|>100$ and contain the location of the resonance in the quasi-2D condensate. In the insets (a) and (b) $f_{2 \mathrm{D}}(-\lambda)$ is plotted at fixed magnetic field and fixed frequency, respectively, as indicated by the lines (a) and (b). At a confinement frequency of $\omega_{z}$ $=0.4 \mathrm{MHz}$ [inset (b)], the Feshbach resonance is shifted by $-16.5 \mathrm{G}$, as shown by the arrow.

mensions disappears. Lee et al. in Ref. [12] show that in the limit of zero temperature in a Bose-Einstein Condensate (BEC) the relevant matrix element of the 2D many-body $T$ matrix $T_{M B}$ is to a good approximation equal to the analytic continuation of the two-body $T$ matrix $T_{2 b}(E)=\left(\hbar^{2} / m\right) f_{2 \mathrm{D}}(E), \quad$ given by $\left\langle 0\left|T_{M B}(E=0)\right| 0\right\rangle$ $=\left\langle 0\left|T_{2 b}(-\lambda)\right| 0\right\rangle$, where $\lambda$ is the chemical potential of the Bose gas. By this procedure, the $i \pi$ term in expression (4) disappears, so that a sharp resonance is expected in a BoseEinstein condensed gas at low temperatures. Formulas (4) and (5) imply that the resonance position in the quasi-2D condensate $B_{0}^{2 \mathrm{D}}$ is different from $B_{0}$. The shift $B_{0}^{2 \mathrm{D}}-B_{0}$ depends on the chemical potential and diverges for $\lambda$ $=\varepsilon^{*}\left(\omega_{z}\right)=C \hbar \omega_{z} / \pi \exp \left[\sqrt{2 \pi} \ell_{z} / a_{b g}\right]$, which is the energy of the weakly bound 2D state if only the background scattering is taken into account $[10,11]$. At this value of the scattering energy, also the width of the resonance in a BEC diverges. For the thermal gas, there are no coherent many-body effects and $\left|f_{2 \mathrm{D}}(E)\right|^{2}$ [expression (4)] is the relevant quantity, for example, in the calculation of the rethermalization rates.

The scattering amplitude in the neighborhood of the Feshbach resonance at $B_{0}=154.9 \mathrm{G}$ in a ${ }^{85} \mathrm{Rb}$ condensate (experimentally explored in Ref. [13]) is shown in Fig. 1 as a function of the confinement frequency and the magnetic field. The resonance parameters are $\Delta B=11.9 \mathrm{G}, a_{b g}$ $=-380 a_{0}$, and $\Delta \mu=-2.23 \mu_{B}[13,14]$. The chemical potential was fixed at $\lambda / \hbar=5 \mathrm{kHz}$. The white regions in Fig. 1 correspond to $|f(-\lambda)|>100$ and indicate the location of the resonance. The divergence of the shift at $\varepsilon^{*}\left(\omega_{z}\right)=\lambda$ can 


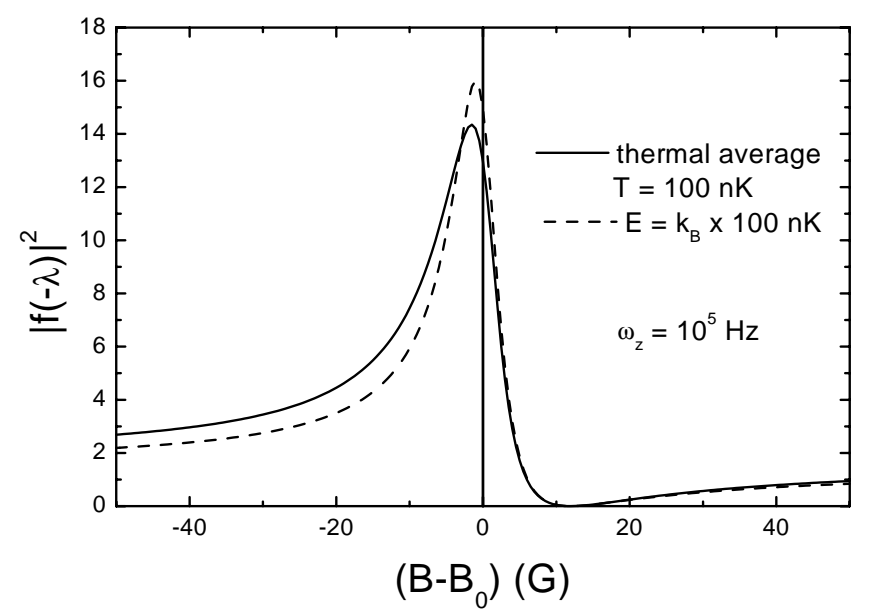

FIG. 2. The modulus square of the scattering amplitude $|f(E)|^{2}$ for a quasi-2D ${ }^{85} \mathrm{Rb}$ thermal cloud is shown for $T=100 \mathrm{nK}$ close to the Feshbach resonance at $B_{0}=154.9 \mathrm{G}$ for and $\omega_{z}=10^{5} \mathrm{~Hz}$. Results are shown with (full line) and without (dashed line) taking thermal broadening into account. In both cases, the divergence of the scattering amplitude has disappeared due to the $2 \mathrm{D}$ character of the gas.

clearly be seen. Inset (b) of Fig. 1 shows the scattering amplitude $f_{2 \mathrm{D}}(-\lambda)$ for condensate atoms at $\omega_{z}=0.4 \mathrm{MHz}$, as a function of magnetic field. For this value of $\omega_{z}$, we find a shift of $B_{0}^{2 \mathrm{D}}-B_{0}=-16.5 \mathrm{G}$, indicated by an arrow in inset (b).

In experiments, it is the radial confinement and the number of particles that are controlled, rather than the chemical potential. The chemical potential for a homogeneous system is equal to $\lambda=\left\langle 0\left|T_{M B}(E=0)\right| 0\right\rangle n_{b}$, with $n_{b}$ the density of bosons and accordingly has to be determined selfconsistently. When the chemical potential is determined selfconsistently in the Thomas-Fermi approximation, again we find that the resonance is shifted. But to the left of the resonance no self-consistent solution for $\lambda$ can be found. In this region we expect the interactions to become attractive, and the absence of a self-consistent solution can be linked to the instability occurring in condensates with attractive interactions.

In Fig. 2, $\left|f_{2 \mathrm{D}}(E)\right|^{2}$, the squared modulus of the scattering amplitude for the thermal cloud, is plotted for $E / k_{B}$ $=100 \mathrm{nK}$. For the thermal gas the maximum value of the scattering amplitude is bounded.

\section{2D CONTACT SCATTERING MODEL}

To avoid having to take into account the details of the real interaction, it is convenient to introduce a $2 \mathrm{D}$ model with an effective contact potential that has the same scattering properties as the real interatomic potential in the quasi-2D system. This effective potential can then be used to tackle manybody problems such as the superfluidity of a two-component fermion gas. Kokkelmans et al. have performed such a study for the 3D case [6]. They introduced a molecular degree of freedom and showed that the model of atoms and molecules that are converted into each other leads to a scattering length of the Feshbach type. We construct an analogous model to describe resonant scattering in a quasi-2D gas. Our model 2D Hamiltonian for the scattering of two different particles 1 and 2 (bosons or fermions) takes the form

$$
\begin{aligned}
\hat{H}= & -\frac{\hbar^{2}}{2} \int d^{2} \mathbf{r}\left[\hat{\psi}_{1}^{\dagger}(\mathbf{r}) \frac{\nabla^{2}}{m_{1}} \hat{\psi}_{1}(\mathbf{r})+\hat{\psi}_{2}^{\dagger}(\mathbf{r}) \frac{\nabla^{2}}{m_{2}} \hat{\psi}_{2}(\mathbf{r})\right] \\
& +V^{P} \int d^{2} \mathbf{r} \hat{\psi}_{1}^{\dagger}(\mathbf{r}) \hat{\psi}_{2}^{\dagger}(\mathbf{r}) \hat{\psi}_{2}(\mathbf{r}) \hat{\psi}_{1}(\mathbf{r}) \\
& +\int d^{2} \mathbf{r} \hat{\psi}_{m}^{\dagger}(\mathbf{r})\left(\frac{-\hbar^{2} \nabla^{2}}{2 m_{m}}+\nu\right) \hat{\psi}_{m}(\mathbf{r}) \\
& +\left[g_{m} \int d^{2} \mathbf{r} \hat{\psi}_{m}^{\dagger}(\mathbf{r}) \hat{\psi}_{2}(\mathbf{r}) \hat{\psi}_{1}(\mathbf{r})+\text { H.c }\right],
\end{aligned}
$$

with $\nu$ the binding energy of the molecule and $g_{m}$ is the parameter related to the formation of a molecule out of two atoms. $V^{P}$ is the interaction far from resonance and $\hat{\psi}_{1}, \hat{\psi}_{2}$, and $\hat{\psi}_{m}$ annihilate the atoms 1,2 , and molecules, respectively. We solve the scattering problem for two atoms in the center of mass frame along the lines of Ref. [6] and we find for the scattering amplitude

$$
\begin{aligned}
f_{2 \mathrm{D}}(\mathbf{k}, \mathbf{p})=2 \sqrt{2 \pi}\left\{\frac{2 \sqrt{2 \pi}\left(\frac{\hbar^{2} k^{2}}{2 m_{\mathrm{red}}}-\nu\right)}{\frac{2 m_{\mathrm{red}}}{\hbar^{2}} V^{P}\left(\frac{\hbar^{2} k^{2}}{2 m_{\mathrm{red}}}-\nu\right)+\left|g_{m}\right|^{2} \frac{2 m_{\mathrm{red}}}{\hbar^{2}}}\right. \\
\left.+\frac{1}{\sqrt{2 \pi}}\left(\ln \left[\frac{K_{c}^{2}}{k^{2}}-1\right]+i \pi\right)\right\}^{-1},
\end{aligned}
$$

which is independent of p. $K_{c}$ is a momentum cutoff, introduced to keep the integrals over $\mathbf{p}$ finite. Expression (7) has the same structure as Eq. (4). Comparing both formulas, using Eq. (5), gives the relation between the physical quantities and the model parameters. With the notation $\chi=2 \Delta B \Delta \mu a_{b g}^{2} m_{\text {red }} / \hbar^{2}$, we find

$$
V^{P}=\frac{1}{1-\chi} \frac{\sqrt{2 \pi} \hbar^{2}}{m_{\mathrm{red}}} \frac{a_{b g}}{\ell_{z}}
$$

for the nonresonant interaction. The molecular binding energy is

$$
\nu=\left(B-B_{0}\right) \Delta \mu /(1-\chi)
$$

and the coupling strength is given by

$$
\left|g_{m}\right|^{2}=V^{P}(\Delta B \Delta \mu+\chi \nu) .
$$

From the comparison of Eqs. (4) and (7), it follows that the cutoff momentum $K_{c}$ is related to the tight confinement har- 
monic oscillator length by $K_{c}=\sqrt{C /\left(\pi \ell_{z}^{2}\right)}$. The righthandside of expression (10) can be negative for some values of $\chi$ and $B$. Because the description in terms of atoms and molecules describes the physics close to the resonance, we need certainly that for $B=B_{0}$ the RHS of Eq. (10) is positive. From the theory of Ref. [9] it follows that $a_{b g} \Delta B \Delta \mu$ $>0$, so our model (6) is applicable only for $\chi<1$. For the case of ${ }^{85} \mathrm{Rb}$ around $B_{0}=154.9 \mathrm{G}$, we find that $\chi=-126.3$ $<1$ so that the model is applicable. In contrast with the 3D case, the parameters $V^{P}$ and $g_{m}$ in 2D case do not depend on the cutoff momentum and need no renormalization, but the cutoff momentum itself is fixed and depends on the tight confinement frequency. Thus, formulas (8), (9), and (10), together with the knowledge of the experimental quantities $a_{b g}, B_{0}, \Delta B$, and $\Delta \mu$ from the 3D Feshbach resonance, fix unambiguously the parameters of the model Hamiltonian (6). This allows us to describe a quasi-2D mixture of gases that are tightly confined in the axial direction by a harmonic potential with characteristic length $\ell_{z}$. As a first check of the model we have found that far from resonance this contact model with a finite cutoff reproduces the critical temperature for superfluidity derived in Ref. [15].

\section{CONCLUSIONS}

The scattering of cold neutral atoms that are strongly confined in one spatial direction is studied analytically close to a Feshbach resonance. We find that there exists a confinementinduced shift in the position of the Feshbach resonance as a function of the magnetic field, and show that for a ${ }^{85} \mathrm{Rb}$ condensate this shift is experimentally detectable. We also find that the Feshbach resonance in the confined thermal gas is damped, in the sense that the scattering amplitude cannot diverge. Based on the present analysis of Feshbach scattering in a quasi-2D gas, we set up a Friedberg-Lee-like model system with an effective contact interaction, suitable for the study of resonant superfluidity in confined gases. Exact relations are derived linking the model parameters to experimentally measurable quantities.

Two of the authors (M.W. and J.T.) are supported financially by the Fund for Scientific Research-Flanders (Fonds voor Wetenschappelijk Onderzoek-Vlaanderen). This research has been supported financially by the FWO-V Project Nos. G.0435.03, G.0306.00, the W.O.G. Project No. WO.025.99N and the GOA BOF UA 2000, IUAP.
[1] M. Holland et al., Phys. Rev. Lett. 87, 120406 (2001); K.M. O'Hara et al., Science 298, 2179 (2002).

[2] M. Greiner et al., Nature (London) 415, 39 (2002).

[3] F.S. Cataliotti et al., Science 293, 843 (2001).

[4] S. Burger et al., Europhys. Lett. 57, 1 (2002).

[5] C. Fort et al., Phys. Rev. Lett. 90, 140405 (2003).

[6] S. Kokkelmans et al., Phys. Rev. A 65, 053617 (2002).

[7] R. Friedberg and T.D. Lee, Phys. Rev. B 40, 6745 (1989).

[8] E.L. Bolda, E. Tiesinga, and P.S. Julienne, Phys. Rev. A 66, 013403 (2002).

[9] A.J. Moerdijk, B.J. Verhaar, and A. Axelsson, Phys. Rev. A 51,
4852 (1995).

[10] D.S. Petrov, M. Holzmann, and G.V. Shlyapnikov, Phys. Rev. Lett. 84, 2551 (2000).

[11] D.S. Petrov and G.V. Shlyapnikov, Phys. Rev. A 64, 012706 (2001).

[12] M.D. Lee et al., Phys. Rev. A 65, 043617 (2002).

[13] J.L. Roberts et al., Phys. Rev. A 64, 024702 (2001).

[14] S. Kokkelmans and M.J. Holland, Phys. Rev. Lett. 89, 180401 (2002).

[15] D.S. Petrov, M.A. Baranov, and G.V. Shlyapnikov, Phys. Rev. A 67, 031601 (2003). 